ARTICLE

DOI: $10.1038 / 541467-018-06878-8$

OPEN

\title{
ZZ-dependent regulation of p62/SQSTM1 in autophagy
}

Yi Zhang (10 1, Su Ran Mun (D) ${ }^{2}$, Juan F. Linares ${ }^{3}$, JaeWoo Ahn ${ }^{1}$, Christina G. Towers ${ }^{1}$, Chang Hoon Ji Brent E. Fitzwalter ${ }^{1}$, Michael R. Holden', Wenyi Mi (1) ${ }^{4}$, Xiaobing Shi ${ }^{4}{ }^{4}$, Jorge Moscat ${ }^{3}$, Andrew Thorburn? Maria T. Diaz-Meco ${ }^{3}$, Yong Tae Kwon ${ }^{2,5}$ \& Tatiana G. Kutateladze (i) ${ }^{1}$

Autophagic receptor p62 is a critical mediator of cell detoxification, stress response, and metabolic programs and is commonly deregulated in human diseases. The diverse functions of p62 arise from its ability to interact with a large set of ligands, such as arginylated (Nt-R) substrates. Here, we describe the structural mechanism for selective recognition of Nt-R by the $\mathrm{ZZ}$ domain of $\mathrm{p} 62$ (p62 zz). We show that binding of $p 62_{z z}$ to Nt-R substrates stimulates p62 aggregation and macroautophagy and is required for autophagic targeting of p62. p62 is essential for mTORC1 activation in response to arginine, but it is not a direct sensor of free arginine in the mTORC1 pathway. We identified a regulatory linker ( $R L$ ) region in p62 that binds $p 62_{z z}$ in vitro and may modulate p62 function. Our findings shed new light on the mechanistic and functional significance of the major cytosolic adaptor protein p62 in two fundamental signaling pathways.

\footnotetext{
${ }^{1}$ Department of Pharmacology, University of Colorado School of Medicine, Aurora, CO 80045, USA. ${ }^{2}$ Protein Metabolism Medical Research Center and Department of Biomedical Sciences, College of Medicine, Seoul National University, Seoul 03080, Republic of Korea. ${ }^{3}$ Cancer Metabolism and Signaling Networks Program, Sanford Burnham Prebys Medical Discovery Institute, La Jolla, CA 92037, USA. ${ }^{4}$ Center for Epigenetics, Van Andel Research Institute, Grand Rapids, MI 49503, USA. 5 Protech Inc., Yongeon 103 Daehangno, Jongno-gu, Seoul 110-799, Republic of Korea. These authors contributed equally: Yi Zhang, Su Ran Mun. Correspondence and requests for materials should be addressed to Y.T.K. (email: yok5@snu.ac.kr)

or to T.G.K. (email: tatiana.kutateladze@ucdenver.edu)
} 
S equestosome 1 (SQSTM1 or p62) mediates cell proliferation, survival, and death through multiple signaling programs, including autophagy and metabolism. This versatile protein adaptor functions as a signaling hub capable of recruiting diverse binding partners and is known to be misregulated in cancer and neurodegenerative disorders ${ }^{1-5}$. New studies of selective protein degradation reveal a critical role of p62 in the autophagic proteolytic cascade responsible for sequestration of toxic, aggregate-prone cargo proteins ${ }^{6-8}$. Following binding to the ubiquitinated cargos, p62 undergoes oligomerization and delivers the cargo aggregates to the autophagosome via interacting with the autophagosomal membrane protein LC3. The sequestered cargo is subsequently degraded by lysosomal enzymes when the autophagosome fuses with a lysosome.

The autophagosomal degradation pathway involves the $\mathrm{N}$ end rule-dependent sensing of a degradation signal, named $\mathrm{N}$-degron ${ }^{9,10}$. The primary determinant of $\mathrm{N}$-degron is a destabilizing amino-terminal residue that can be produced via proteolytic cleavage of the substrate or enzymatically added and is targeted by a protein effector, or N-recognin ${ }^{9,11}$. One of the major and widespread $\mathrm{N}$-degrons in eukaryotes is the aminoterminal arginine residue (Nt-R). Arg-tRNA transferases enzymatically add arginine to the protein sequence starting with either aspartic acid or glutamic acid and the resultant arginylated sequence is recognized by the UBR-box domain of UBRs ${ }^{12-15}$. Recent studies have identified the ZZ domain of p62 (p62 $\mathrm{ZZ})$ as a new Nt-R recognin ${ }^{6-8}$, however, the molecular mechanism by which it interacts with the degron and the biological importance of this interaction remain unclear.

p62 is also an important modulator of the nutrient sensor mTORC $1^{16,17}$. It functions as a scaffold protein that recruits components of the mTORC1 signaling machinery to a specific subcellular location ${ }^{18}$. Free amino acids, lysine and arginine particularly, activate the mTORC1 response and promote $\mathrm{p} 62$ phosphorylation $^{19}$. Binding of p62 to the E3-ubiquitin ligase TRAF6 then leads to polyubiquitination of the mTORC1 complex subunit and facilitates its translocation to the lysosome ${ }^{18,19}$.

Structurally, p62 contains six functional motifs: an N-terminal PKC-binding PB1 (Phox and Bemlp) domain, the central $\mathrm{ZZ}$ and TB modules, a LC3-interacting region (LIR), a Keap1binding region (KIR), and the C-terminal ubiquitin associated (UBA) domain (Fig. 1a). Various cellular stresses have been shown to stimulate p62 oligomerization, mediated by the PB1 domain and the region C-terminal to $\mathrm{PB} 1^{8,20,21}$. The PB1mediated oligomerization is essential for cargo collection and aggregation by p62 and the delivery of p62-cargo complexes to the autophagosome ${ }^{6,21}$.

Here, we report the structural basis underlying specific recognition of Nt-R substrates by the ZZ domain of p62. We demonstrate that this interaction promotes p62 aggregation and is necessary for macroautophagy. We propose a novel mechanism for p62 autoregulation in which binding of the $\mathrm{ZZ}$ domain to the internal p62 sequence may play a role in regulating p62 function.

\section{Results and Discussion}

Structural basis for the Nt-R recognition by $\mathrm{p} 62_{\mathrm{ZZ}}$. To gain insight into the molecular mechanism by which p62 recognizes $\mathrm{Nt}-\mathrm{R}$ substrates, we determined the atomic-resolution structure of the p62 $\mathrm{zz}$-RE complex. We designed and crystallized a chimeric construct containing the Arg-Glu sequence fused via a short linker to the $\mathrm{N}$-terminus of the $\mathrm{ZZ}$ domain of $\mathrm{p} 62$ (aa 120-171) (hereafter referred to as RE-ZZ) (Figs. 1b, c). In the structure, two RE-ZZ molecules form a complex in which the $\mathrm{RE}$ residues of one molecule are bound by the $\mathrm{ZZ}$ domain of another molecule, whereas the linker residues are flexible and have no contact with either ZZ domain (Supplementary Figure 1). An extensive network of salt bridges and hydrogen bonds stabilizes the p62 $\mathrm{zz}-\mathrm{RE}$ complex (Figs. $1 \mathrm{~b}, \mathrm{c}$ ). The $\mathrm{Nt}-\mathrm{R}$ is bound in a highly negatively charged groove lined with the residues D129, N132, D147, and D149 of p62. The guanidinium moiety of Arg1 is constrained by a salt bridge with the carboxyl group of D129 and hydrogen bonds with the oxygen atom of the carboxamide group of N132. Notably, the a-amino-terminal $\mathrm{NH}_{3}{ }^{+}$group of Arg1 is bound via a set of intermolecular contacts, including salt bridges and hydrogen bonds with the carboxyl groups of D129 and D149 and the backbone carbonyl group of I127. In addition, the carbonyl group of Arg1 is hydrogen bonded to the backbone amide of I127. The complex is further stabilized through the interactions involving degron's Glu2. The side chain carboxyl group of Glu2 forms a salt bridge with the guanidinium moiety of R139, which in turn is restrained through electrostatic and hydrogen bonding contacts with D149, whereas the backbone amide of Glu2 donates a hydrogen bond to the carboxyl group of D147 (Fig. 1c). Remarkably, the same residues R139, D149, and D129 that are implicated in the intermolecular interactions with the Nt-R substrate, also interact with each other, yielding a unique arrangement of intertwined contacts that define the $\mathrm{p} 62_{\mathrm{ZZ}}$ selectivity towards the RE sequence (Figs. 1c, 2a).

The selectivity of $\mathbf{p} 62_{\mathrm{zz}}$. To establish the selectivity, we tested p $62_{\mathrm{ZZ}}$ in ${ }^{1} \mathrm{H},{ }^{15} \mathrm{~N}$ heteronuclear single quantum coherence (HSQC) titration experiments (Figs. 1d, e, 2b). As shown in Fig. 1d, the REEE peptide induced large chemical shift perturbations (CSPs) in uniformly ${ }^{15} \mathrm{~N}$-labeled $\mathrm{p} 62_{\mathrm{ZZ}}$. CSPs were in slow-to-intermediate exchange regime, indicative of a tight interaction. A number of amide crosspeaks of the $\mathrm{p} 62_{\mathrm{ZZ}}$ apo-state disappeared upon addition of the peptide and another set of resonances corresponding to the bound state appeared (Fig. 1d). In contrast, no CSPs were detected when Ac-REEE peptide in which the a-amino-terminal $\mathrm{NH}_{3}{ }^{+}$group of Arg1 is blocked by acetylation was titrated in, pointing to the critical role of the free amino terminus in the interaction (Fig. 1e, left panel). However, the free amino terminus alone was insufficient, as the binding was mostly eliminated when Arg1 was substituted with alanine in the AEEE peptide (Fig. 1e, middle panel). Titration of the RAEE peptide in which Glu2 is replaced with alanine led to CSPs that were in the intermediate, and no longer in the slow exchange regime, indicating a decrease in the binding activity of p62 $\mathrm{zz}$ (Fig. 1e, right panel). In agreement, dissociation constant $\left(K_{\mathrm{d}}\right)$ measured by microscale thermophoresis (MST) for the interaction of p62 $\mathrm{zz}$ with REEE was found to be $5.6 \mu \mathrm{M}$, whereas binding of the RAEE peptide was reduced $\left(K_{\mathrm{d}}=14 \mu \mathrm{M}\right)$ (Figs. 2ce). The binding affinities were corroborated by intrinsic tyrosine fluorescence measurements (Figs. 2d, e). Altogether, these results indicate that an $\mathrm{N}$-terminally unmodified arginine in the first position is required for the degron sequence to be recognized by p62 $\mathrm{zZ}$, and the presence of a glutamic acid next to the arginine further enhances this interaction.

A contribution of the RE-binding site residues to the binding energetics was assessed through mutating D129, D147, and D149 of $\mathrm{p} 62_{\mathrm{Zz}}$ individually to lysine and examining the mutant proteins by nuclear magnetic resonance (NMR) (Fig. 2b). The absence of CSPs in NMR samples of the three mutants upon titration with REEE indicated that each of these $\mathrm{p} 62_{\mathrm{ZZ}}$ residues is required for the interaction. Furthermore, binding capabilities of $\mathrm{N} 132 \mathrm{~A}$ and $\mathrm{R} 139 \mathrm{~A}$ mutants of $\mathrm{p} 62_{\mathrm{zz}}$ were also substantially compromised, implying that proper coordination of the Arg1 and Glu2 side chains is essential (Fig. 2e and Supplementary Figure 2). 
a

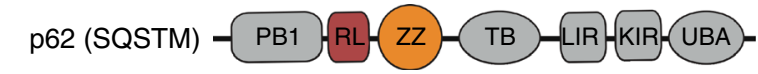

b
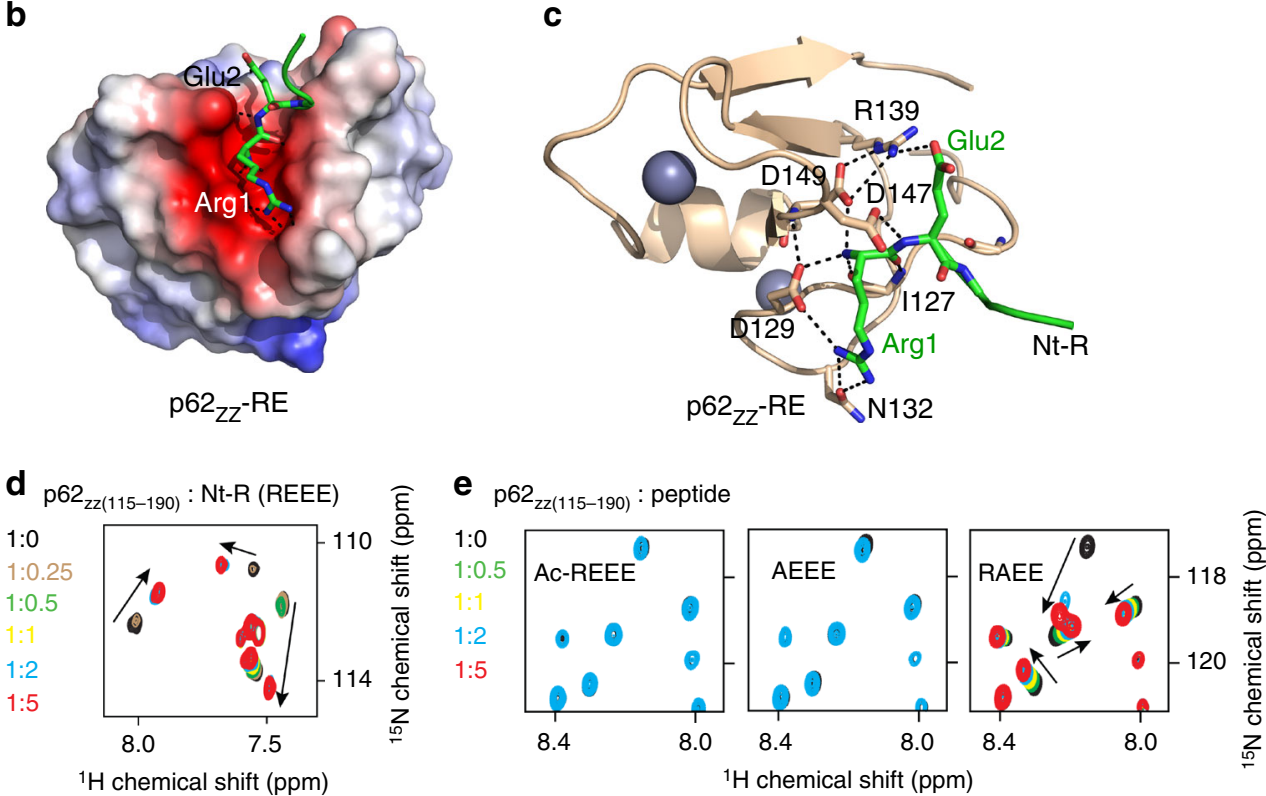

Fig. 1 p62zz is a recognin of Nt-R. a p62/SQSTM domain architecture. b Electrostatic surface potential of p62zz colored blue and red for positive and negative charges, respectively. The bound Nt-R substrate (residues RE) are shown in stick. c A ribbon diagram of the crystal structure of p62 zz (wheat) in complex with the Nt-R substrate (green). Dashed lines indicate hydrogen bonds and salt bridges. d Superimposed ${ }^{1} \mathrm{H}_{1}{ }^{15} \mathrm{~N} \mathrm{HSQC}$ spectra of p62 $\mathrm{zZ}$ (115-190) collected while the REEE peptide was titrated in. Spectra are color coded according to the protein:peptide molar ratio. e Superimposed ${ }^{1} \mathrm{H},{ }^{15} \mathrm{~N} \mathrm{HSQC}$ spectra of $\mathrm{p} 62_{\mathrm{zz}}$ (115-190) collected upon titration with the indicated 4-mer peptides. Spectra are color coded according to the protein:peptide molar ratio

Much like the isolated $\mathrm{p} 62_{\mathrm{zZ}}$ domain, full-length $\mathrm{p} 62$ ectopically expressed in HEK293 cells robustly recognized arginylated substrates in pulldown assays. We tested $\mathrm{X}-\mathrm{ns} \mathrm{P} 4$ peptides, corresponding to the arginylated $(\mathrm{X}=\mathrm{Arg})$ or glycinylated $(\mathrm{X}=\mathrm{Gly})$ amino-terminal sequence of the $\mathrm{N}$-end rule substrate Sindbis virus. Whereas wild-type p62 bound to the arginylated $\mathrm{R}$-nsP4 peptide, it did not associate with the glycinylated G-nsP4 peptide (Fig. 2f). In contrast, none of the D129K, D147K, and D149K point mutant $\mathrm{p} 62$ proteins were able to recognize R-nsP4. Similarly, p62 associated with the peptides that correspond to the native substrates of p62-arginylated amino-terminal region of the ER-residing molecular chaperone $\mathrm{BiP}$ and arginylated amino-terminal region of CRT, but not with the respective peptides containing intrinsic glutamate or valine at the N-terminus (Figs. 2g, h).

To date, $\mathrm{p} 62_{\mathrm{zZ}}$ and the UBR-box are the only known receptors for type-I Nt-degron. Although both domains employ a set of negatively charged residues to engage the Nt- $\mathrm{R}$ degron, their structural topologies and the Nt-degron recognition mechanisms are different (Fig. 2i). Furthermore, p62 $\mathrm{zz}$ and the UBR-box differ in their preferences for the residue at position 2 of the Nt-degron. Although $\mathrm{p} 62_{\mathrm{Zz}}$ prefers a negatively charged residue (Glu2, as in the physiological substrate $\mathrm{BiP}$ ), the UBR domain shows preference for a hydrophobic residue due to a hydrophobic pocket located nearby ${ }^{14}$. This difference can help to explain the selective recognition and fine-tuned sorting of substrates toward proteasomal and autophagic degradation pathways.

Binding of $\mathrm{p}^{2} \mathrm{zz}$ to arginylated substrates induces autophagy. To define the biological significance of Nt-R recognition by $\mathrm{p} 62 \mathrm{zZ}$ in vivo, we monitored p62 puncta formation in p62-/- mouse embryonic fibroblast (MEF) cells, in which $p 62^{f / f}$ was deleted using the CRE recombinase, expressing WT protein and loss-of- function mutant D129K. The cells were treated with or without XIE62-1004, a recently developed small molecule ligand of $\mathrm{p} 62_{\mathrm{ZZ}}$ that induces p62 polymerization and facilitates collection of autophagic cargoes such as misfolded proteins and their aggregates $^{8}$. The XIE62-1004 treatment readily induced formation of cytosolic puncta positive for p62 in MEF cells expressing WT p62, whereas cells expressing D129K mutant were incapable of responding to the treatment (Figs. 3a, b). We then examined XIE62-1004-induced autophagosome formation in cells expressing WT p62 or the D129K, D147K and D149K mutants. After treatment with the autophagic blocker hydroxychloroquine (HCQ), immunoblotting analysis of LC3 showed that XIE62-1004 strongly promotes autophagosome biogenesis in cells expressing WT p62 (Fig. 3c). Immunoblotting assays also showed that XIE62-1004 promotes the lipidation of LC3 in WT MEFs, whereas p62-/- MEFs did not respond to XIE62-1004 (Fig. 3d). Furthermore, the efficacy of XIE62-1004 to induce LC3 lipidation was restored when recombinant p62 was stably expressed in p62-/- MEFs, but not when 62 mutants D129K, D147K, and D149K were expressed (Fig. 3d and Supplementary Figure 3a). Collectively, these data indicate that the binding of $\mathrm{p} 62_{\mathrm{zz}}$ to arginylated substrates induces autophagy and is also required for p62 puncta formation in cells.

We further characterized autophagic function of $\mathrm{p} 62_{\mathrm{ZZ}}$ through investigating different subtypes of autophagy, such as macroautophagy and mitophagy. To quantitatively monitor functional autophagy, particularly autolysosome formation in cells, an mCherry-GFP-LC3 tandem construct was stably expressed, and the acidic quenching of green fluorescent protein (GFP) signal relative to the stable expression of mCherry was measured by flow cytometry 22 (Fig. 4a). In agreement with immunoblotting analysis of LC3, Earle's balanced salts solution (EBSS)-induced starvation caused a dramatic increase in the percent of cells undergoing 
a

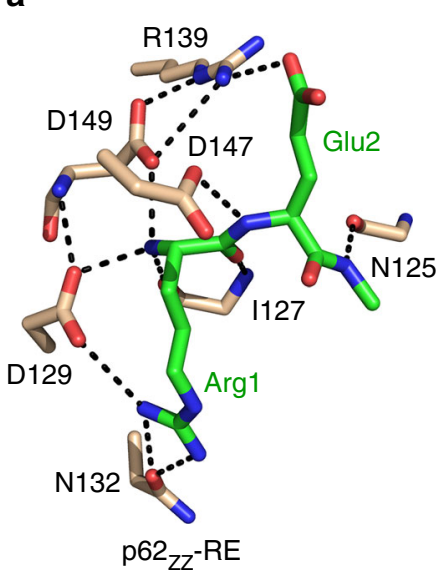

b
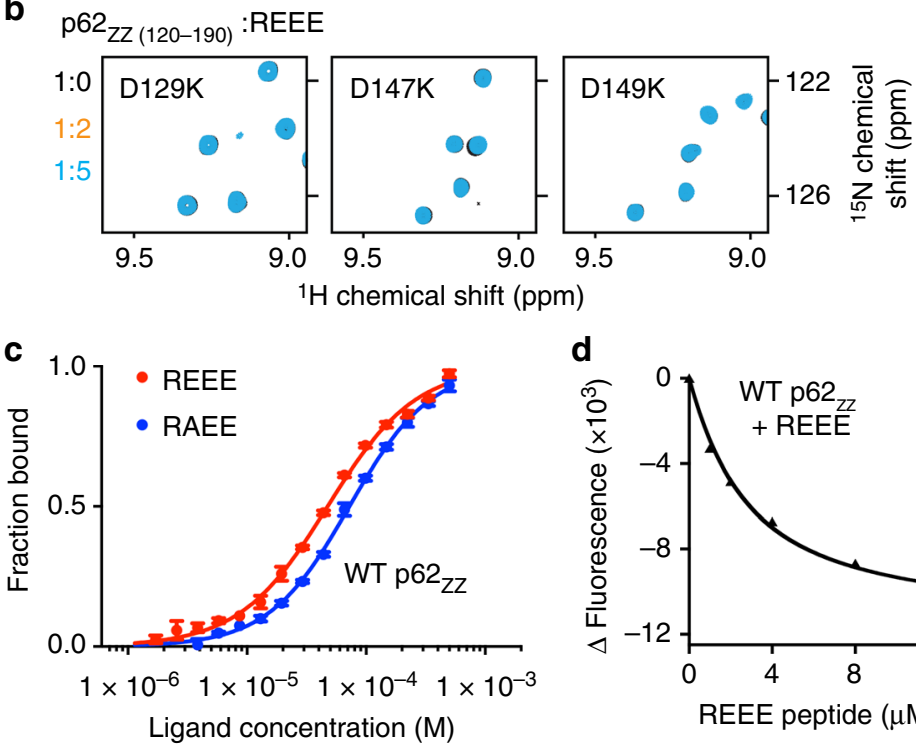
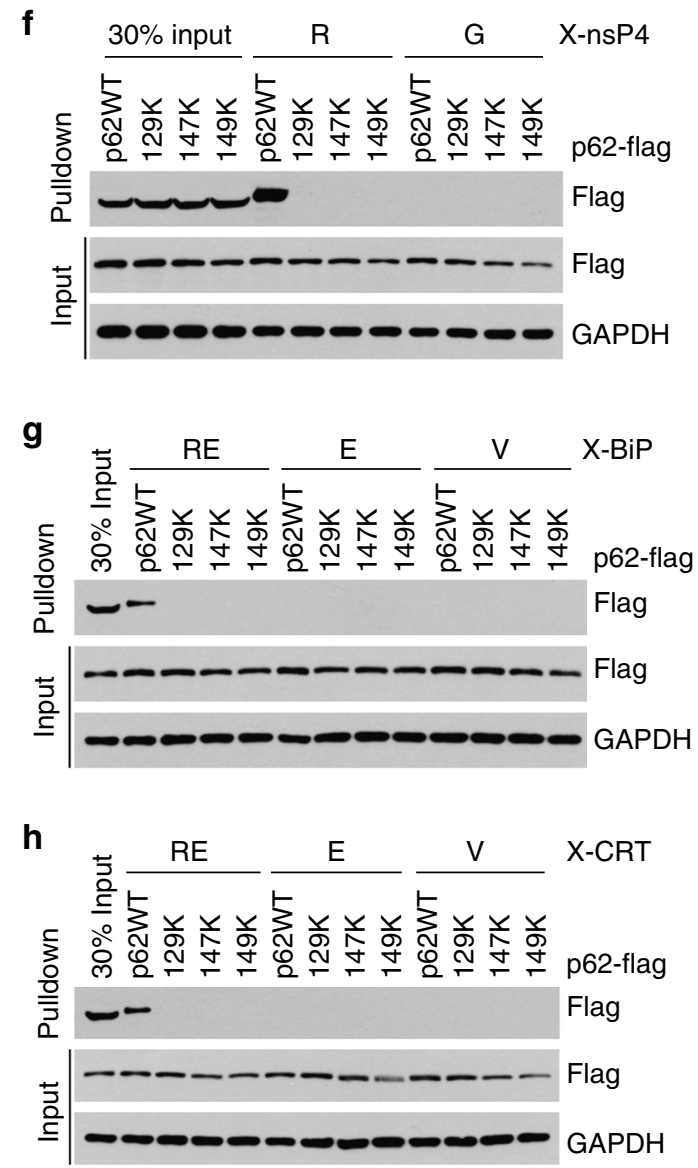

e

\begin{tabular}{ccc}
\hline $\mathrm{p} 62_{\mathrm{zz}}$ & Ligand & $K_{\mathrm{d}}(\mu \mathrm{M})$ \\
\hline WT & NH2-REEE & $1.9 \pm 0.4^{\mathrm{a}}$ \\
& & $5.6 \pm 0.4^{\mathrm{b}}$
\end{tabular}

$\begin{array}{ccc}\text { WT } & \text { Ac-REEE } & \mathrm{nb} \\ \text { WT } & \text { NH2-AEEE } & n b \\ \text { WT } & \text { NH2-RAEE } & 3.2 \pm 0.5^{\mathrm{a}} \\ & & 14 \pm 1^{\mathrm{b}}\end{array}$

D129K NH2-REEE nb

D147K NH2-REEE nb

D149K NH2-REEE nb

D139K NH2-REEE $\quad 1300 \pm 300^{\circ}$

N132A NH2-REEE $\quad 250 \pm 80^{\circ}$

i

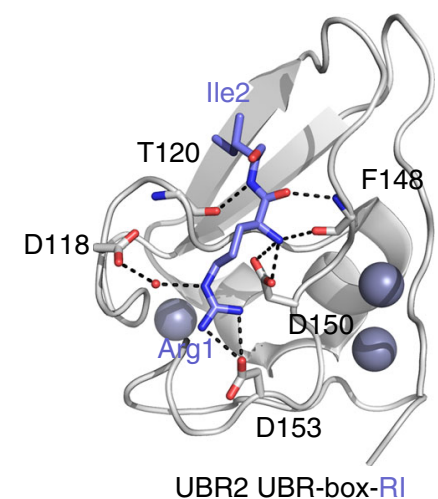

Fig. 2 Molecular basis for the specific targeting of Nt-R by p62 ${ }^{Z Z}$. a A zoom-in view of the Nt-R degron binding site. b Superimposed ${ }^{1} \mathrm{H}^{15} \mathrm{~N} H S Q C$ spectra of p62zz (120-190) collected upon titration with the REEE peptide. Spectra are color coded according to the protein:peptide molar ratio. c, d Representative binding curves used to determine the $K_{d}$ values by MST (c) or tyrosine fluorescence (d). Error bar in c represents s.d. in triplicate measurements. e Binding affinities of p62zz for the indicated peptides measured by MST (a, using p62zz (115-190) ) or tyrosine fluorescence $\left({ }^{b}\right.$, using p62 zz (120-171) ) or NMR ( ${ }^{c}$, using p62zz (115-190)). Errors represent s.d. calculated from curve fitting (a, c) or duplicate midarguments (b). f-h Pulldown assays of wild-type or mutated p62Flag expressed in HEK293 cells using X-nsP4 (f), X-BiP (g), and X-CRT (h) peptides. Uncropped blots are shown in Supplementary Figure 7. i A ribbon diagram of the structure of UBR-box of UBR2 (gray) in complex with Nt-R degron (blue, PDB 3NYN) 
a

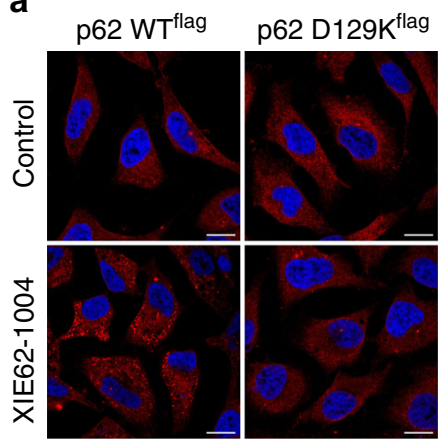

b

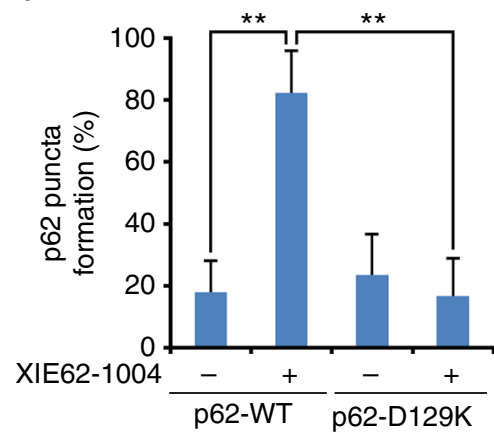

C

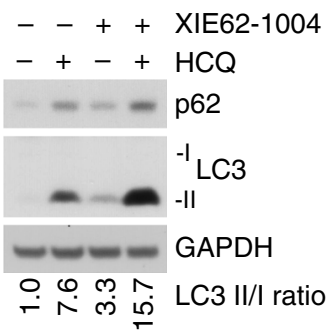

d

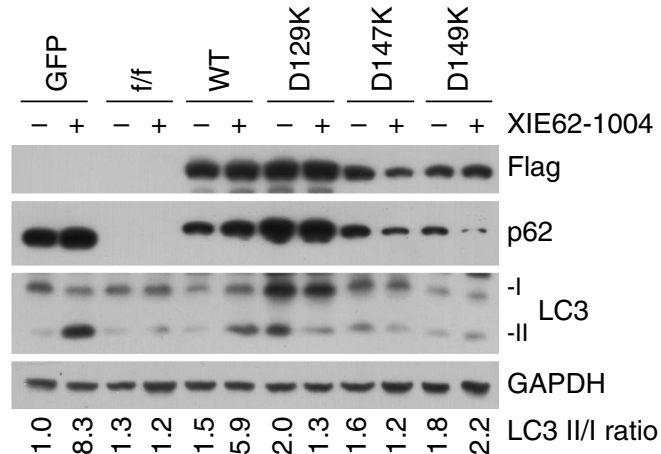

Fig. 3 p62zz mediates stress-induced autophagy. a Mouse embryonic fibroblasts expressing recombinant wild-type or D129K point mutant p62-flag were treated with XIE62-1004 ( $5 \mu \mathrm{M}, 6 \mathrm{~h})$ or DMSO negative control, followed by immunostaining analysis. Scale bar, $10 \mu \mathrm{m}$. b Quantification of a ( $p$ calculated by two-tailed Student's $t$-test; data shown as mean \pm S.D. of three independent experiments each with $n=30$ cells; $\left.{ }^{\star \star} p<0.01\right)$. c Immunoblotting assay of endogenous p62-expressing mouse embryonic fibroblasts treated with XIE62-1004 $(5 \mu \mathrm{M}, 6 \mathrm{~h})$ in the presence or absence of hydroxychloroquine $(10 \mu \mathrm{M}$, $24 \mathrm{~h}$ ) for autophagic flux assay. $\mathbf{d}$ Western blot analysis of mouse $\mathrm{p} 62^{\mathrm{fl} / \mathrm{fl}}$ embryonic fibroblasts infected with GFP or CRE adenoviruses were reconstituted with retroviruses expressing WT Flag-p62 or point mutants of Flag-p62 treated with/without XIE62-1004 (5 $\mu$ M, 6 h). Ratio between LC3-II and LC3-I quantified by densitometry using ImageJ

autophagy, which was indicated by a decrease in GFP expression (Fig. 4b). In starved conditions, addback of WT p62 increased the percent of cells undergoing autophagy, however, rescue with D129K or D147K mutant of p62 failed to do so (Figs. 4c, d). Together, these results showed that induction of autophagy depends on the functional $\mathrm{ZZ}$ domain. Further analysis revealed that 162 affects macroautophagy but does not cause a significant increase in the percent of cells undergoing mitophagy, the form of autophagy that degrades mitochondria (Fig. 4e). Overall, these data suggest that $\mathrm{p} 62_{\mathrm{ZZ}}$ is necessary for efficient macroautophagy induced by EBSS, whereas p62 is dispensable in mitophagy.

We next examined the role of $\mathrm{p} 62_{\mathrm{ZZ}}$ in selective autophagy using cells under misfolded protein stress, induced by proteasome inhibitor MG132. Immunoblotting analysis showed that p62-/MEFs failed to properly induce the lipidation of LC3 as compared with $+/+$ MEFs when the cells were treated with MG132 (Fig. 4f and Supplementary Figure 3b). Notably, stable expression of WT p62 allowed p62-/- MEFs to induce autophagy under the same condition (Fig. 4f). Consistent with the aforementioned results, mutation of either D129, D147, or D149 abolished the ability of cells to induce LC3 lipidation and autophagosome formation (Fig. $4 \mathrm{~g}$ and Supplementary Figure 3b). These data point to the critical role of $\mathrm{p} 62_{\mathrm{ZZ}}-\mathrm{Nt}-\mathrm{R}$ interaction in autophagy under proteasomal inhibition.

p62 $\mathrm{zZ}$ mediates p62 aggregation in vitro. p62-dependent selective autophagy involves polymerization of p62 in response to stress stimuli, including protein misfolding and oxidative stress $6,8,20,23,24$, and in vitro, recombinant p62 forms aggregates within a few days ${ }^{25}$. Both the PB1 domain and the linker connecting $\mathrm{PB} 1$ and $\mathrm{ZZ}$ have been implicated in polymerization ${ }^{21,26,27}$. PB1 was shown to form homo- and hetero-oligomers ${ }^{23,26,27}$, whereas the linker region is needed to form disulfide-coupled aggregated conjugates via Cys1138,20 and/or tubular structures, as demonstrated by cryo-EM studies ${ }^{21}$. To clarify the role of $\mathrm{p} 62_{\mathrm{Zz}}$ in $\mathrm{p} 62$ polymerization, we performed in vitro aggregation assays in which extracts from MEFs expressing p62 were treated with or without dipeptides and reactions were resolved by non-reducing sodium dodecyl sulfate-polyacrylamide gel electrophoresis (SDS-PAGE) (Supplementary Figure 4). In the absence of stimulation, WT p62 existed in a monomer- $250 \mathrm{kDa}$ oligomers equilibrium, in support of the findings that this polymerization step is PB1 domain dependent $^{8}$ (Supplementary Figure $4 \mathrm{a}, \mathrm{b}$ ). The p62 $\mathrm{zz}$ mutants (D129K, D147K, and D149K) also formed $\sim 250 \mathrm{kDa}$ oligomers, indicating that this step is $\mathrm{ZZ}$ domain independent. Consistent with previous reports ${ }^{8}$, stimulation with the Arg-Ala peptide but not by the reverse Ala-Arg peptide induced p62 aggregation, which was disulfide bond formation dependent (Supplementary Figure 4a, c). However, the D129K, D147K, and D149K mutants of p62 that do not bind to Nt-R showed substantially reduced aggregation in the presence of either peptide, demonstrating that autophagic p62 aggregation depends on both the functional ZZ domain and stimulation with Arg-Ala (Supplementary Figure 4c). These results together with the autophagosome formation data suggest that functional $\mathrm{p} 62_{\mathrm{Zz}}$ is necessary for mediating autophagic aggregation of $\mathrm{p} 62$ in vitro and in vivo.

p62 is not a direct arginine sensor in mTORC1 pathway. To determine whether the amino-acid arginine, which is abundant 
a

\begin{tabular}{|c|c|c|}
\hline \multicolumn{3}{|c|}{ Autophagy } \\
\hline mCherry & eGFP & LC3 \\
\hline \multicolumn{3}{|c|}{ Mitophagy } \\
\hline mCherry & eGFP & Fis1 \\
\hline \multicolumn{2}{|c|}{$\begin{array}{c}\text { Autophagosome } \\
\text { neutral } p H \text { environment }\end{array}$} & $\square+\square=\square$ \\
\hline \multicolumn{2}{|c|}{$\begin{array}{l}\text { Autolysosome } \\
\text { low pH environment }\end{array}$} & $\square+\square=\square$ \\
\hline
\end{tabular}

b
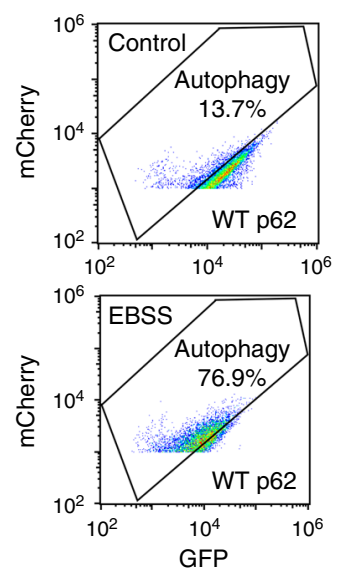

GFP

e
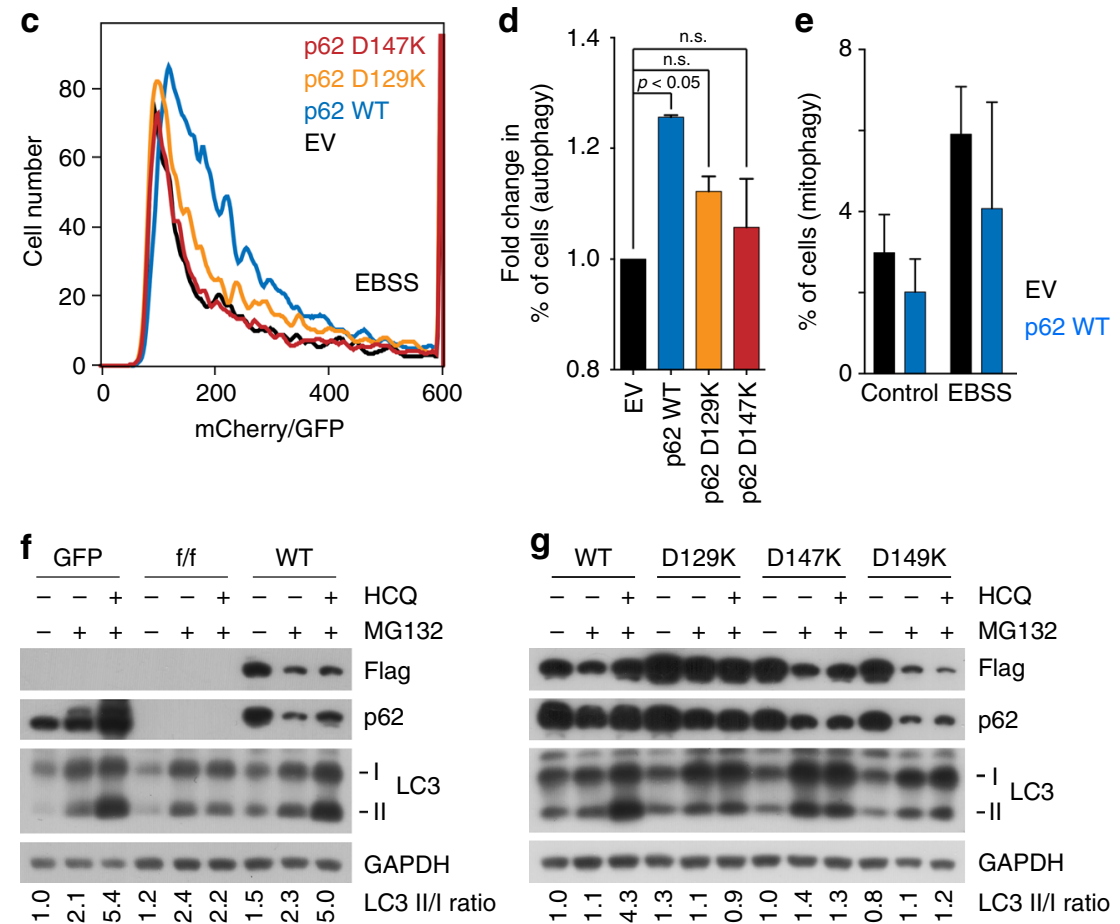

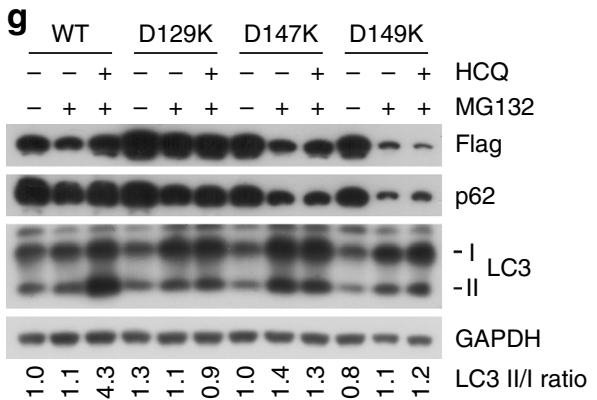

Fig. 4 p62zz is necessary for efficient EBSS-induced macroautophagy. a Schematic of LC3 tandem and Fis-tandem flux assay. b An example of the LC3 tandem flow cytometry assay under basal conditions or starved (5\% serum media in 95\% EBSS media) conditions in p62 WT MEFs, where a decrease in GFP expression can be observed and quantified to measure the percent of cells undergoing autophagy. c Number of cells undergoing autophagy $24 \mathrm{~h}$ after starvation in 5\% serum media in EBSS: measured by the decreased ratio of mCherry/GFP via flow cytometry in p62-/- MEFS with stable expression of an empty vector, WT p62, D129K p62 or D147K p62 and stable expression of mCherry-GFP-LC3 tandem (representative figure of two experiments).

d Quantification of (c), where the percent of cells undergoing autophagy was normalized to the p62-/- condition. One-way ANOVA was performed on two independent experiments. e Normalized percent of cells undergoing mitophagy measured by the decreased ratio of mCherry/GFP via flow cytometry in p62-/- MEFS with stable expression of an empty vector, WT p62, D129K p62, or D147K p62 and stable expression of mCherry-GFP-Fis1 tandem. Error bar in $\mathbf{d}$ and $\mathbf{e}$ represents s.e.m with $N$ of 2 . $\mathbf{f}$ Western blot analysis of mouse p62 $2^{\mathrm{fl} / \mathrm{fl}}$ embryonic fibroblasts infected with GFP or CRE adenoviruses were reconstituted with retroviruses expressing WT Flag-p62 or point mutants of Flag-p62 treated with/without MG132 (0.2 $\mu \mathrm{M}, 24 \mathrm{~h})$ in the presence or absence of hydroxychloroquine $(10 \mu \mathrm{M}, 24 \mathrm{~h})$. Ratio between LC3-II and LC3-I quantified by densitometry using ImageJ. $\mathbf{g}$ Similar to $\mathbf{f}$ but with mouse embryonic fibroblasts expressing recombinant wild-type p62-flag or indicated point mutants

in the cytosol, could be a substrate for p62 $\mathrm{ZZ}$, we employed NMR titration experiments. As shown in Fig. 5a, a free arginine binds to p62 $\mathrm{zZ}$, but free glutamic acid, leucine, or glutamine do not. To elucidate the mechanistic basis for the arginine association, we obtained the crystal structure of $\mathrm{p} 62_{\mathrm{ZZ}}$ in complex with arginine (Fig. 5b). Structural comparison of $\mathrm{p} 62_{\mathrm{ZZ}}-\mathrm{RE}$ and $\mathrm{p} 62_{\mathrm{ZZ}}$-arginine complexes reveals that the intermolecular contacts constraining the side chain and the $\alpha$-amino-terminal $\mathrm{NH}_{3}{ }^{+}$group of Arg1 are conserved in both complexes, however, D147 and R139 no longer contribute to the interaction with free arginine.
Given the ability to bind arginine and its central role as a positive regulator of the mTORC1 pathway, we reasoned that p62 could affect the capacity of mTORC1 to respond to arginine. Indeed, p62-/- cells, starved of arginine (Fig. 5c) or amino acids (Fig. 5d), showed a severe inhibition of the mTORC1 activation in response to arginine, suggesting that p62 is necessary for arginine-mediated mTORC1 activation. To determine whether the activation of $\mathrm{mTORC} 1$ by arginine requires the arginine-binding capacity of p62, we reconstituted p62-/- cells with retroviruses expressing p62 WT or the p62 mutants that are 
a

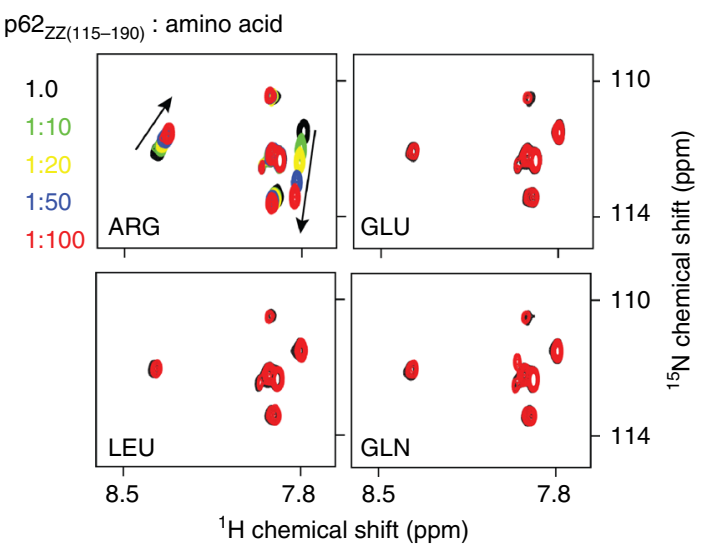

C
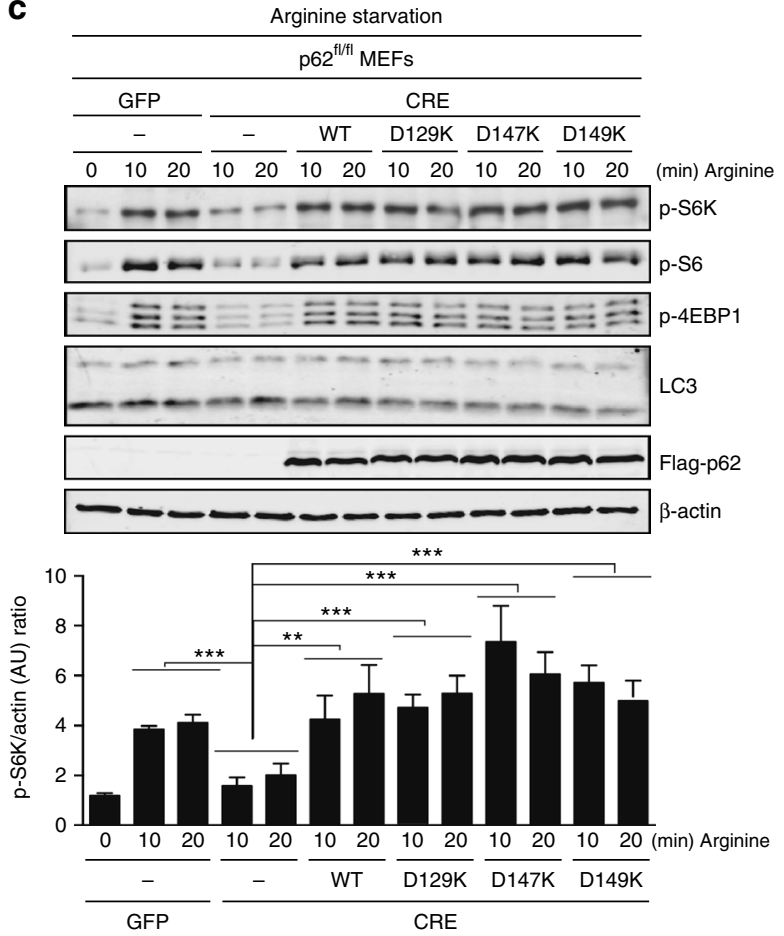

b

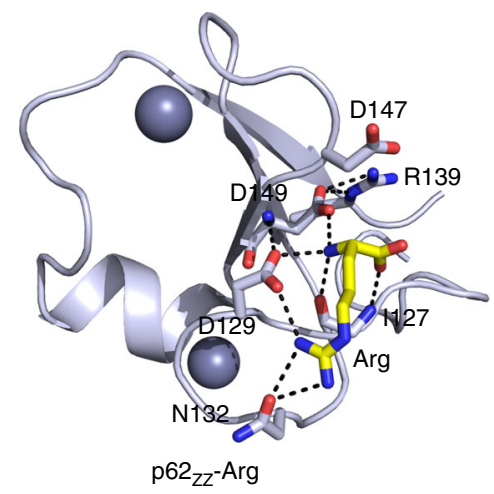

d
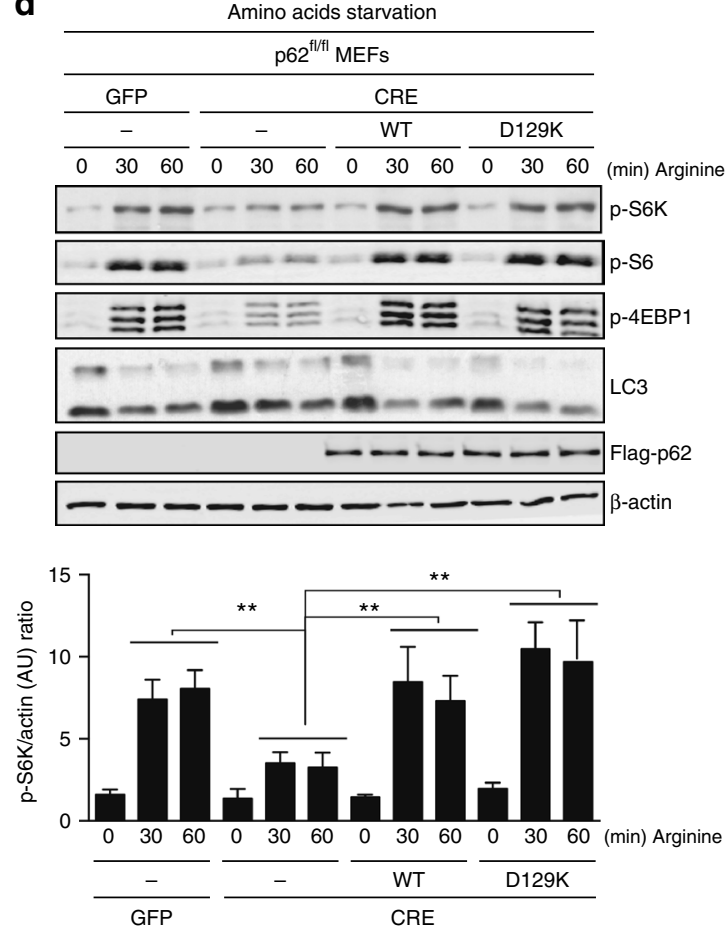

Fig. 5 p62 is not a direct arginine sensor in mTORC1 pathway. a Superimposed ${ }^{1} \mathrm{H},{ }^{15} \mathrm{~N}$ HSQC spectra of p62 $\mathrm{zz}$ (115-190) collected upon titration with the indicated free amino acids. Spectra are color coded according to the protein:ligand molar ratio. $\mathbf{b}$ A ribbon diagram of the crystal structure of p62 $2 z$ (wheat) in complex with the amino-acid arginine (yellow). c Mouse p62 ${ }^{\mathrm{fl} / \mathrm{fl}}$ embryonic fibroblasts infected with GFP or CRE adenoviruses were reconstituted with retroviruses expressing Flag-p62 WT, D129K, D147K, or D149K. Cells were deprived of arginine for $4 \mathrm{~h}$ and then stimulated with arginine for the indicated durations. Cell lysates were analyzed for the levels of the specified proteins. Graphs represent p-S6K/actin ratio as measured by densitometry using Image studio software ( $n=3$ independent experiments). d p $62^{\mathrm{fl} / \mathrm{fl}}$ MEFs cells infected with GFP or CRE adenoviruses and stably expressing Flag-p62 WT or D129K, were deprived of amino acids for $4 \mathrm{~h}$ and then stimulated with arginine for the indicated durations. Cell lysates were analyzed for the levels of the specified proteins. Graphs represent p-S6K/actin ratio as measured by densitometry using Image studio software ( $n=3$ independent experiments). Twoway ANOVA test ${ }^{* *} p<0.01,{ }^{\star \star *} p<0.001$. Error bar in $\mathbf{c}$ and $\mathbf{d}$ represents s.e.m. with $N$ of 3

unable to bind arginine (D129K, D147K, or D149K) and analyzed the arginine-mediated mTORC1 activation under arginine (Fig. 5c) or amino acids (Fig. 5d) starvation. We found that overexpression of the p62 mutants, impaired in arginine binding, rescued the mTORC1 activation almost at the same level as WT p62. These data demonstrate that activation of the mTORC1 pathway by arginine is largely independent of the argininebinding capacity of $\mathrm{p} 62$. Interestingly, the autophagy pathway was activated after starvation as indicated by notable lipidation of LC3 (compare LC3-II bands in Figs. 5c, d). Re-addition of arginine or amino acids had little effect (if not inhibiting) on autophagy under tested conditions. Taken together, our findings suggest that unlike arginylated substrates, a free arginine is incapable of inducing autophagy.

p62 $\mathrm{zZ}$ interacts with RL. Modeling peptide mimetics in the REbinding site of $\mathrm{p} 62_{\mathrm{ZZ}}$ suggested that the sequence containing a tandem of positively charged lysine or arginine residues followed by a negatively charged glutamate or aspartate can mimic the $\mathrm{Nt}$ RE sequence (Fig. 6). Intriguingly, the 20-residue linker connecting the PB1 and ZZ domains in p62 contains two such motifs, i.e., KKE (aa 102-104 of p62) and RRD (aa 106-108 of p62) (Fig. 6b). To determine whether p62 $\mathrm{zZ}$ can bind to the internal 
a p62 $_{\text {zz(115-190) }}$ : amino acid
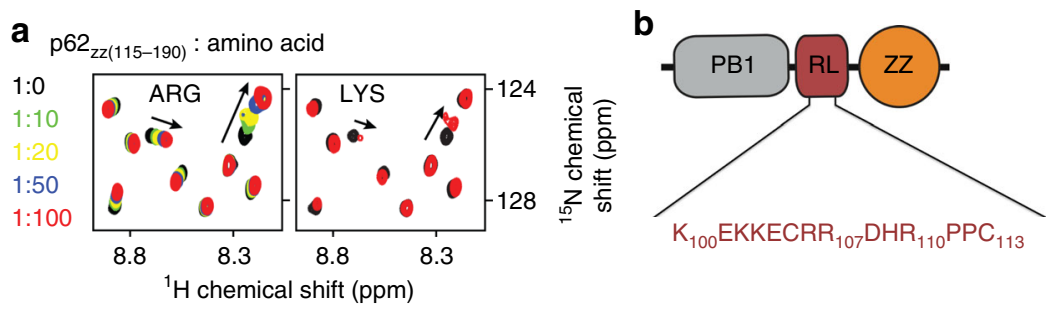

$$
\text { C }
$$
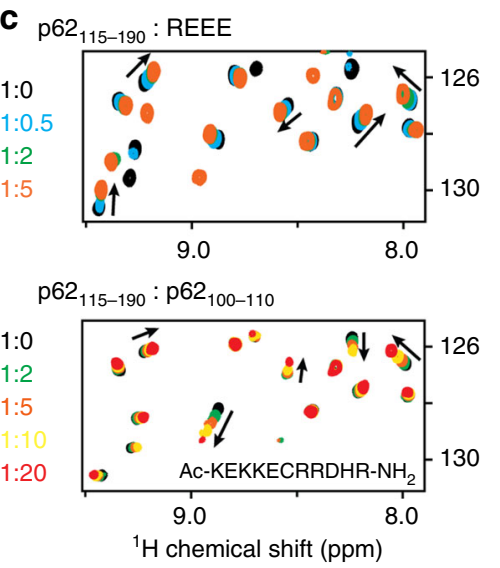

e

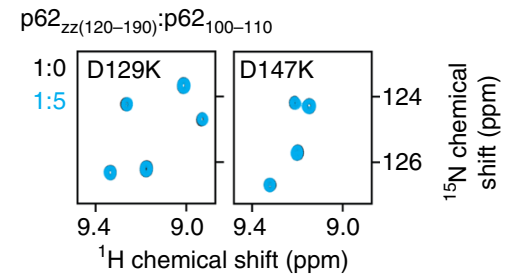

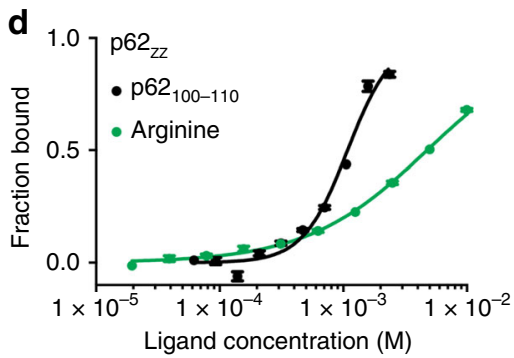

f

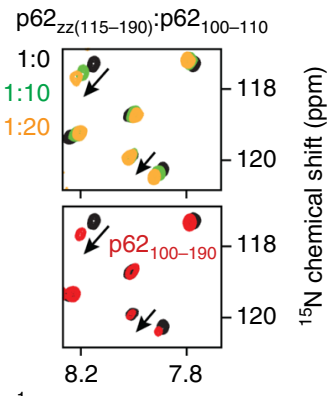

${ }^{1} \mathrm{H}$ chemical shift $(\mathrm{ppm})$

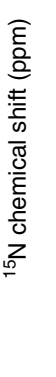

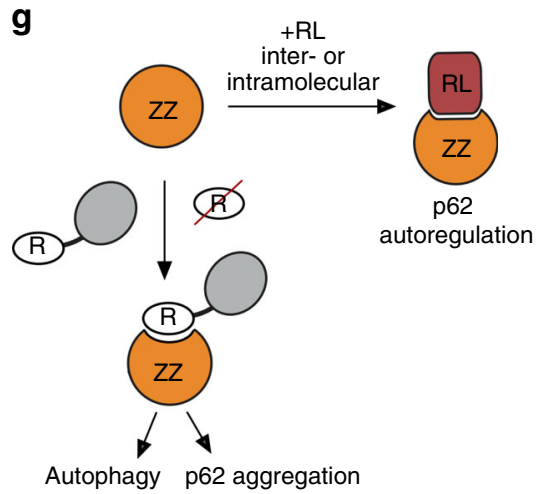

Fig. 6 p62zz interacts with RL. a Superimposed ${ }^{1} \mathrm{H}_{1}^{15} \mathrm{~N}$ HSQC spectra of p62zz (115-190) collected upon titration with the indicated amino acids showing that p 62 zz has a weak binding activity for lysine. Spectra are color coded according to the protein:ligand molar ratio. $\mathbf{b}$ Schematic representation of the p62 $\mathrm{N}$ terminal region containing PB1 and ZZ domains. c Superimposed ${ }^{1} \mathrm{H}, 15 \mathrm{~N} \mathrm{HSQC}$ spectra of $\mathrm{p} 62_{\mathrm{ZZ}}$ (115-190) collected upon titration with the indicated peptides. Spectra are color coded according to the protein:peptide molar ratio. d Binding curves used to determine binding affinities of p62zz by MST. Error bar represents s.d. from triplicate measurements. e Superimposed ${ }^{1} \mathrm{H}_{1}^{15} \mathrm{~N} \mathrm{HSQC}$ spectra of $\mathrm{p} 62_{\mathrm{zz}}$ (120-190) collected upon titration with the RL peptide. Spectra are color coded according to the protein:peptide molar ratio. $f$ Superimposed ${ }^{1} \mathrm{H}_{1}^{15} \mathrm{~N} \mathrm{HSQC}$ spectra of p62 $\mathrm{zz}$ (115-190) collected upon titration with the p62 $\mathrm{zZ}$ (100-110) peptide (top) and superimposed ${ }^{1} \mathrm{H}_{1}^{15} \mathrm{~N} \mathrm{HSQC}$ spectra of p62 $\mathrm{zZ}$ (115-190) and p62 $\mathrm{RL}-\mathrm{ZZ}$ (100-190). $\mathrm{g}$ A model of p62 function and autoregulation. The $\mathrm{ZZ}$ domain interacts with the RL region either intra- or inter- molecularly. Binding of the ZZ domain to Nt-R degron but not arginine is necessary for p62 aggregation and autophagy

sequence, we probed association of p62 $\mathrm{zZ}$ with the $\mathrm{K}_{100}$ EKKECRRDHR $_{110}\left(\mathrm{p} 62_{100-110}\right)$ peptide by NMR and MST. Large CSPs in $\mathrm{p} 62_{\mathrm{ZZ}}$ upon gradual addition of the p62 $100-110$ peptide indicated direct interaction (Fig. 6c). Although patterns of CSPs induced by REEE and p62 $100-110$ peptides were not identical, a similar set of amides was perturbed in either titration, implying that the same binding site of $\mathrm{p} 62_{\mathrm{zz}}$ accommodates both peptides. In contrast, the $\mathrm{D} 129 \mathrm{~K}$ and $\mathrm{D} 147 \mathrm{~K}$ mutants of $\mathrm{p} 62_{\mathrm{ZZ}}$, which are defective in binding to Nt-R, did not interact with RL (Fig. 6e), corroborating the notion that the RL-binding site of p62 $\mathrm{ZZ}$ at least partially overlaps with the Nt-R-binding site of this domain. MST measurements yielded a $K_{\mathrm{d}}$ of $320 \mu \mathrm{M}$ for the interaction between $\mathrm{p} 62_{\mathrm{Zz}}$ and the p62 $62_{100-110}$ peptide, however, we note that this interaction must be tighter in the physiologically relevant condition, because $\mathrm{RL}$ and $\mathrm{ZZ}$ are physically linked in p62 (Fig. 6d). Indeed, NMR amide resonances of the construct 
containing RL natively linked to $\mathrm{p} 62_{\mathrm{ZZ}}$ suggested formation of the stable RL-p62 $\mathrm{zz}$ complex (Fig. 6f and Supplementary Figure 5).

In conclusion, in this study we have elucidated the molecular mechanism for selective recognition of the arginylated substrates by $\mathrm{p} 62_{\mathrm{ZZ}}$. We show that binding of $\mathrm{p} 62_{\mathrm{ZZ}}$ to Nt-R degron induces autophagy and that this interaction is required for p62 puncta formation in cells. We further demonstrate that p62 is necessary for arginine-mediated mTORC1 activation but $\mathrm{p} 62$ does not serve as a direct arginine sensor. We identified a regulatory linker (RL) between the PB1 domain and the $\mathrm{ZZ}$ domain that is recognized by $162_{\mathrm{ZZ}}$ in vitro. Although further studies are necessary to fully understand the interplay between the multiple binding partners of $\mathrm{p} 62_{\mathrm{ZZ}}$, the data presented here suggest that recognition of the internal p62 sequence by $\mathrm{p} 62_{\mathrm{ZZ}}$ could modulate p62 activities (Fig. $6 \mathrm{~g}$ ). Our results show that $\mathrm{p} 62_{\mathrm{Zz}}$ binds tighter to RL than to free arginine amino acid $\left(K_{\mathrm{d}}=1.5 \mathrm{mM}\right.$, Fig. $\left.6 \mathrm{~d}\right)$, however, weaker to Nt-R degron. This difference in binding affinities can be critical in directing p62 toward the distinct autophagic and mTORC1 signaling pathways. The autoregulation involving RL could in turn be modulated by 62 phosphorylation, particularly of the p62 $\mathrm{zz}$ residues Tyr148 and Ser170. Future studies will be required to test this idea and to establish whether the $\mathrm{p} 62_{\mathrm{ZZ}}-\mathrm{RL}$ interaction occurs in an intermolecular or intramolecular manner. Furthermore, it will be important to investigate the role of the disease-relevant mutations in $\mathrm{p} 62_{\mathrm{ZZ}}$ and $\mathrm{RL}$, for example, $\mathrm{R} 107 \mathrm{~W}$ and $\mathrm{D} 129 \mathrm{~N}$, identified in patients with amyotrophic lateral sclerosis ${ }^{28}$.

\section{Methods}

Protein expression and purification. The human p62 ZZ domain (aa 100-190, aa $115-190$, and aa 120-190) was cloned into a pGEX 6p-1 vector and expressed in BL21 (DE3) RIL cells. The p62 ZZ domain (aa 120-171) and RE-linked ZZ domain (aa 120-171 of p62, following the sequence RELGS) were cloned into a pCIOX vector with an N-terminal His-tag and Ulp1 cleavage site. Primers are listed in Supplementary Figure 6. Protein production was induced with $0.2 \mathrm{mM}$ isopropyl $\beta$ D-1-thiogalactopyranoside (IPTG) overnight at $16^{\circ} \mathrm{C}$ in Luria broth (LB) or minimal media (M9) supplemented with ${ }^{15} \mathrm{NH}_{4} \mathrm{Cl}$ and $0.05 \mathrm{mM} \mathrm{ZnCl}_{2}$. The glutathione S-transferase (GST)-tagged ZZ proteins were purified on glutathione Sepharose $4 \mathrm{~B}$ beads (GE Healthcare) in $20 \mathrm{mM}$ Tris- $\mathrm{HCl}$ ( $\mathrm{pH} 7.0$ ) buffer, supplemented with $100 \mathrm{mM} \mathrm{NaCl}$ and $5 \mathrm{mM}$ DTT. The GST tag was cleaved with PreScission protease overnight at $4{ }^{\circ} \mathrm{C}$. The His-tagged proteins were purified on $\mathrm{Ni}$-NTA beads (Qiagen) in $20 \mathrm{mM}$ Tris- $\mathrm{HCl}$ ( $\mathrm{pH}$ 7.5) buffer, supplemented with $300 \mathrm{mM} \mathrm{NaCl}$ and $10 \mathrm{mM} \beta$-mercaptoethanol. The His tag was cleaved overnight at $4{ }^{\circ} \mathrm{C}$ with PreScission or Ulp1 protease. Unlabeled and ${ }^{15} \mathrm{~N}$-labeled proteins were further purified by size exclusion chromatography and concentrated in Millipore concentrators. All mutants were generated by site-directed mutagenesis using the Stratagene QuikChange mutagenesis protocol, grown, and purified as WT proteins.

NMR experiments. NMR experiments were carried out at $298 \mathrm{~K}$ on Varian INOVA 600 and $900 \mathrm{MHz}$ spectrometers. NMR samples contained $0.1 \mathrm{mM}$ uniformly ${ }^{15} \mathrm{~N}$-labeled WT or mutated p62 $\mathrm{zz}$ in $20 \mathrm{mM}$ Tris- $\mathrm{HCl}(\mathrm{pH} 7.0)$ buffer supplemented with $100 \mathrm{mM} \mathrm{NaCl}, 5 \mathrm{mM}$ DTT, and $8 \% \mathrm{D}_{2} \mathrm{O}$. Binding was characterized by monitoring CSPs in the proteins induced by peptides (synthesized by SynPeptide or KE BioChem) and amino acids. $K_{\mathrm{d}}$ was determined by applying principal component analysis to the ${ }^{1} \mathrm{H},{ }^{15} \mathrm{~N}$ HSQC titration spectra in TREND ${ }^{29,30}$. Each binding isotherm was fitted using the following equation:

$$
p_{\text {bound }}=\|\mathrm{PC} 1\|=\frac{\left(\left([L]+[P]+K_{d}\right)-\sqrt{\left([L]+[P]+K_{d}\right)^{2}-4[P][L]}\right)}{2[P]}
$$

where $[L]$ is concentration of the ligand, $[P]$ is concentration of $Z Z$, $p_{\text {bound }}$ is the fraction of protein bound to ligand, and $\|\mathrm{PC} 1\|$ is the normalized principal component, obtained by TREND, which indicates the change in the population of the bound state. The errors of the $K_{\mathrm{d}}$ value are the fitting uncertainties from nonlinear least-squares fits using Kaleidagraph.

X-ray crystallography. The RE-linked p62 $\mathrm{zz}$ (aa $120-171$ of $\mathrm{p} 62$, following the sequence RELGS) and p62 $\mathrm{ZZ}$ (aa 120-171) in complex with arginine were crystalized. RE-p62 $\mathrm{ZZ}$ was purified by size exclusion chromatography using a S100 (GE Healthcare) column equilibrated in buffer containing $20 \mathrm{mM}$ Tris-HCl (pH 7.5), $100 \mathrm{mM} \mathrm{NaCl}$ and $1 \mathrm{mM}$ TCEP and concentrated to $1.5-2.5 \mathrm{mg} / \mathrm{mL}$. Crystals were obtained using hanging drop vapor diffusion against an equal volume of the well solution containing $0.1 \mathrm{M}$ BICINE, pH 8.0, 20\% PEG 6000 at $18{ }^{\circ} \mathrm{C}$. Crystals were cryoprotected with the addition of $30 \%$ glycerol to the well solution and the X-ray diffraction data were collected on the UC Denver X-ray crystallography core facility Rigaku Micromax 007 high-frequency microfocus X-ray generator equipped with a Pilatus $200 \mathrm{~K} 2 \mathrm{D}$ area detector. For crystallization of $\mathrm{p} 62_{\mathrm{zz}}$ in complex with arginine, the eluted protein was precipitated by ultracentrifugation and then resolved in buffer containing $0.1 \mathrm{M}$ arginine ( $\mathrm{pH} 7.5$ ) to a final concentration of $20 \mathrm{mg} / \mathrm{mL}$. Crystals were obtained by seeding at $18^{\circ} \mathrm{C}$ in condition containing 0.1 M Bicine (pH 9.0), 14\% PEG 20 K, 4\% 1,4-Dioxane. Data collection was performed at the ALS 4.2.2 beamline, Berkeley. Indexing, integrating, and scaling were processed by HKL $3000^{31}$. The phase solution was found by single-wavelength Anomalous Dispersion method with $\mathrm{Zn}$ anomalous signal or using molecular replacement. Model building and refinement were carried out with $\operatorname{Coot}^{32}$ and Phenix.refine ${ }^{33}$. The final structure was validated with MolProbity. The statistics of the data collection and refinement are summarized in Supplementary Table 1.

Fluorescent MST-binding assay. The MST experiments were performed using a Monolith NT.115 instrument (Nanotemper). All experiments were performed with the purified ZZ domain (aa 115-190) in a buffer containing $10 \mathrm{mM}$ HEPES (pH 7.4), $150 \mathrm{mM} \mathrm{NaCl}$ and $1 \mathrm{mM}$ TCEP. The final concentration of the fluorescein (FAM)-labeled peptide (KE BIOCHEM) was kept at $80 \mathrm{nM}$. Dissociation constants for the interaction between $\mathrm{ZZ}$ with unlabeled peptides (REEE, RAEE, and p62 $100-110)$ and free arginine were measured using a displacement assay in which increasing amount of unlabeled peptides were added into a preformed ZZ:FAM-

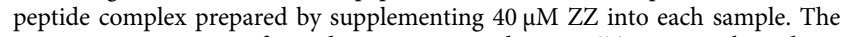
measurements were performed at 50\% LED and 40\% MST power with 3-s laser-on time and 25-s off time. For all measurements, samples were loaded into premium capillaries and 1300-1700 counts were obtained for the fluorescence intensity. The $K_{\mathrm{d}}$ and $\mathrm{IC}_{50}$ values were determined with the MO. Affinity Analysis software (NanoTemper Technologies $\mathrm{GmbH}$ ). The $K_{\mathrm{i}}$ values for unlabeled peptides with $\mathrm{ZZ}$ were determined from the $\mathrm{IC}_{50}$ values observed in the displacement assay and converted by the following equation:

$$
K_{i}=[I]_{50} /\left(\frac{[L]_{50}}{K_{d}}+\frac{[P]_{0}}{K_{d}}+1\right)
$$

where $[I]_{50}$ is the concentration free unlabeled ligand at $50 \%$ binding, $[L]_{50}$ is the concentration of free labeled $\mathrm{H} 3$ peptide at $50 \%$ binding. The $K_{\mathrm{d}}$ value is the dissociation constant of FAM-labeled peptide determined in the direct binding experiment described above. All measurements were done in triplicates.

Fluorescence spectroscopy. Spectra were recorded at $25^{\circ} \mathrm{C}$ on a Fluoromax- 3 spectrofluorometer (HORIBA). The samples containing $1.0 \mu \mathrm{M}$ p62 $\mathrm{ZZ}$ domain (aa $120-171)$ and progressively increasing concentrations of the peptide were excited at $274 \mathrm{~nm}$. Experiments were performed in buffer containing $10 \mathrm{mM}$ HEPES ( $\mathrm{pH}$ 7.4), $150 \mathrm{mM} \mathrm{NaCl}$ and $1 \mathrm{mM}$ TCEP. Emission spectra were recorded over a range of wavelengths between 285 and $315 \mathrm{~nm}$ with a $0.5 \mathrm{~nm}$ step size and a 1 -s integration time and averaged over three scans. The $K_{\mathrm{D}}$ values were determined using a nonlinear least-squares analysis and the equation:

$$
\Delta I=\Delta \mathrm{I}_{\max } \frac{\left(\left([L]+[P]+K_{d}\right)-\sqrt{\left([L]+[P]+K_{d}\right)^{2}-4[P][L]}\right)}{2[P]}
$$

where $[L]$ is the concentration of the peptide, $[P]$ is the concentration of $\mathrm{ZZ}$ domain, $\Delta \mathrm{I}$ is the observed change of signal intensity, and $\Delta I_{\max }$ is the difference in signal intensity of the free and bound states of the $\mathrm{ZZ}$ domain. The $K_{\mathrm{D}}$ value was averaged over three separate experiments, with error calculated as the standard deviation between the runs.

Amino acids and arginine starvation and arginine stimulation. For amino acids starvation, MEFs ${ }^{18}$ were rinsed with phosphate-buffered saline (PBS) and incubated in amino acid-free RPMI (USBiological), supplemented with $10 \%$ dialyzed serum, for $4 \mathrm{~h}$. For arginine starvation, MEFs were rinsed with PBS and incubated with arginine, leucine and lysine-free RPMI (USBiological), supplemented with $0.38 \mathrm{mM}$ of leucine and $0.21 \mathrm{mM}$ of lysine, and $10 \%$ dialyzed serum, for $4 \mathrm{~h}$. Cells were stimulated with $1 \mathrm{mM}$ of arginine for different durations. Cells were rinsed with PBS and immediately lysed with Ripa lysis buffer (1\% Triton, 0.5\% SDS, $10 \mathrm{mM} \beta$-glycerol phosphate, $10 \mathrm{mM}$ pyrophosphate, $40 \mathrm{mM}$ Hepes $\mathrm{pH} 7.4,2.5$ $\mathrm{mM} \mathrm{MgCl}_{2}$ and 1 tablet of EDTA-free protease inhibitor (Roche)). The cell lysate were cleared by centrifugation at $13,000 \mathrm{rpm}$ at $4{ }^{\circ} \mathrm{C}$ in a microcentrifuge for 10 minutes. Cell extracts were denatured by addition of $1 \times$ sample buffer followed by boiling for $5 \mathrm{~min}$, resolved by $8-14 \%$ SDS-PAGE, and then transferred to nitrocellulose-ECL membranes (GE Healthcare).

Measurement of autophagic flux by ratiometric flow cytometry. Cells stably expressing mCherry-GFP-LC3 or mCherry-GFP-Fis ${ }^{34}$ were used for flow cytometric analysis. Briefly, a Gallios 561 (Beckman Coulter) using 488 and $561 \mathrm{nM}$ lasers for green and red fluorophore excitation, respectively, was used to perform 
flow cytometry. The appropriate forward/side scatter profile was used to exclude non-viable cells. Cells undergoing autophagy were defined as those expressing a high mCherry/GFP fluorescence ratio, as delivery by autophagy to the lysosome quenches the GFP signal, but not the mCherry signal. The gates to define what constituted an increased mCherry/GFP fluorescence ratio were set based on cells treated overnight with bafilomycin A1 (10 nM, Sigma-Aldrich B1792; CAS RN: 88899-55-2), a condition that represents cells with little or no autophagic flux. The bottom of the gate for each set of flow cytometry experiments was therefore set at the rightward base of the bafilomycin A1-treated curve with the gate such that no $>5 \%$ of bafilomycin A1-treated cells were included in the gate.

X-peptide pulldown assay. A set of synthetic 12-mer peptides were C-terminally biotin conjugated. The X-nsP4 peptide (X-IFSTIEGRTYK-biotin) has Arg (arginylated) or Gly (stabilized) at the N-terminus.

A set of 10-mer BiP-derived peptides (X-EEDKKEDVG-biotin) and 10-mer CRT peptides (X-EPAVYFKEQ-biotin) bearing N-terminal Arg-Glu (permanently arginylated), Glu (native), or Val (glutamic acid-to-valine mutant) were used. For cross-liking with resin, C-terminally biotin-conjugated peptides were mixed with high capacity streptavidin agarose resin (Thermo, \#20361) with a ratio of $0.5 \mathrm{mg}$ peptide per $1 \mathrm{ml}$ settled resin and incubated on rotator at $4{ }^{\circ} \mathrm{C}$ for overnight. After washing five times with PBS, the peptide-beads conjugates were diluted with PBS at 1:1 ratio. To prepare protein extracts, cells were collected by centrifugation and lysed by freezing and thawing at least ten times in Hypotonic Buffer $(10 \mathrm{mM} \mathrm{KCl}$, $1.5 \mathrm{mM} \mathrm{MgCl}_{2}$, and $10 \mathrm{mM}$ HEPES, pH 7.9) with a protease inhibitor mix (Sigma, P8340). After spinning down with centrifugation in $12,000 \mathrm{rpm}$ at $4^{\circ} \mathrm{C}$ for $20 \mathrm{~min}$, proteins were quantified using BCA protein assay kit (Thermo Scientific, \#23227). Total proteins $(70 \mu \mathrm{g})$ diluted in $250 \mu \mathrm{l}$ Binding Buffer $(0.05 \%$ Tween-20, $10 \%$ glycerol, $0.2 \mathrm{M} \mathrm{KCl}$, and $20 \mathrm{mM}$ HEPES-pH 7.9) were mixed with $50 \mu$ l peptidebead resin and incubated at $4{ }^{\circ} \mathrm{C}$ for $2 \mathrm{~h}$ on a rotator. The protein-bound beads were collected by centrifugation at $5000 \mathrm{rpm}$ for $2 \mathrm{~min}$ and washed five times with binding buffer. The beads were resuspended in $25 \mu \mathrm{SDS}$ sample buffer, heated at $95^{\circ} \mathrm{C}$ for $5 \mathrm{~min}$, and subjected to SDS-PAGE and immunoblotting.

In vitro $\mathbf{p 6 2}$ aggregation assays. Cells were lysed by 10 cycles of freezing and thawing in lysis buffer (50 mM HEPES, pH 7.4, 0.15 M KCl, 0.1\% Nonidet P-40, $10 \%$ glycerol, and a mixture of protease inhibitors and phosphatase inhibitor), followed by centrifugation at $13,000 \times g$ for $20 \mathrm{~min}$ at $4^{\circ} \mathrm{C}$. The protein concentration in supernatant was determined using BCA assay. Total $5 \mu \mathrm{g}$ protein was mixed with $50 \mathrm{mM}$ of Arg-Ala or Ala-Arg dipeptide in the presence of $100 \mu \mathrm{M}$ bestatin at room temperature for $2 \mathrm{~h}$. Non-reducing sampling buffer containing $4 \%$ lithium dodecyl sulfate was added to each sample, heated at $95^{\circ} \mathrm{C}$ for $2 \mathrm{~min}$, and subjected to SDS-PAGE and immunoblotting.

Cell culture and immunoblotting. HEK293 and MEF cell lines were cultured in Dulbecco's modified Eagle's medium (GIBCO) supplemented with $10 \%$ fetal bovine serum (GIBCO) in a 5\% $\mathrm{CO}_{2}$ incubator. For immunoblotting, cells were lysed in SDS-based sample buffer (277.8 mM Tris-HCI, pH 6.8, 4.4\% LDS, $44.4 \%$ (v/v) glycerol) with beta-mercaptoethanol. Using SDS-PAGE, whole-cell lysates were separated and transferred onto polyvinylidene difluoride membranes at $100 \mathrm{~V}$ for $2 \mathrm{~h}$ at $4^{\circ} \mathrm{C}$. Subsequently, the membrane was blocked with $4 \%$ skim milk in PBS solution for $30 \mathrm{~min}$ at room temperature and incubated overnight with primary antibodies, followed by incubation with host-specific horseradish peroxidase (HRP)-conjugated secondary antibodies. The primary antibodies used were: anti-phospho-S6K (Cell Signaling, \#9205), anti-phospho-4EBP1 (Cell Signaling, \#2855), anti-phospho-S6 (Cell Signaling, \#5364), anti-LC3 (Cell Signaling \#4108, Sigma \#L7543, and Novus \#NB100-2220), anti- $\beta$-actin (Sigma, \#A1978 and \#A5441), anti-Flag (Sigma \#F3165 and Sigma \#F1804), anti-p62 (Novus \#H00008878 and Abcam \#56416), and anti-GAPDH (BioWorld, \#AP0063). The secondary antibodies used were: anti-IgG1 (BD Biosciences \#550331, Cell Signaling \#7974 and \#7076), anti-rabbit (Dako, \#E0432), and Alexa fluor488 anti-IgG (Invitrogen \#A11034 and \#11029). Details of antibodies and dilutions are listed in Supplementary Data 1. For signal detection, a mixture of ECL solution (Thermo Fisher Scientific) was applied onto the membrane and captured using X-ray films. Densitometry of band intensity was performed using ImageJ (NIH, Bethesda).

Immunocytochemistry. To observe cellular localization of proteins, cells were cultured on cover slips coated with poly-L-lysine. The cells were fixed with $4 \%$ paraformaldehyde in PBS ( $\mathrm{pH}$ 7.4) for $15 \mathrm{~min}$ at room temperature and washed three times with PBS for $5 \mathrm{~min}$. The cells were permeabilized with $0.5 \%$ Triton X100/PBS solution for $15 \mathrm{~min}$ and washed three times with PBS for $5 \mathrm{~min}$. The cells were blocked with $2 \%$ bovine serum albumin (BSA)/PBS solution for $1 \mathrm{~h}$ at room temperature. After blocking, the cells were incubated overnight at $4{ }^{\circ} \mathrm{C}$ with primary antibody diluted in $2 \% \mathrm{BSA} / \mathrm{PBS}$ solution. Following incubation, the cells were washed three times for 10 min with PBS and incubated with secondary antibody diluted in $2 \%$ BSA/PBS for $30 \mathrm{~min}$ at room temperature. Subsequently, the cover slips were mounted on glass slides using a DAPI-containing mounting medium. Confocal images were taken by laser scanning confocal microscope 510 Meta (Zeiss) and analyzed by Zeiss LSM Image Browser (ver. 4.2.0.121). Subsequently, cells were deemed to exhibit significant colocalization if $>10$ puncta structures of the respective proteins showed association or full colocalization. Quantification results are shown as mean \pm S.D. of three independent experiments.

\section{Data availability}

The atomic coordinates and structure factors of the $\mathrm{p} 62_{\mathrm{Zz}}$ complexes have been deposited in the Protein Data Bank under the accession codes 6MIU and 6MJ7. Other data are available from the corresponding authors upon reasonable request.

Received: 2 May 2018 Accepted: 2 October 2018

Published online: 22 October 2018

\section{References}

1. Duran, A. et al. p62/SQSTM1 by binding to vitamin D receptor inhibits hepatic stellate cell activity, fibrosis, and liver cancer. Cancer Cell 30, 595-609 (2016)

2. Moscat, J. \& Diaz-Meco M. T. p62 at the crossroads of autophagy, apoptosis, and cancer. Cell 137, 1001-1004 (2009).

3. Todoric, J. et al. Stress-activated NRF2-MDM2 cascade controls neoplastic progression in pancreas. Cancer Cell 32, 824-839 e828 (2017).

4. Mathew, R. et al. Autophagy suppresses tumorigenesis through elimination of p62. Cell 137, 1062-1075 (2009).

5. Ramesh Babu, J. et al. Genetic inactivation of p62 leads to accumulation of hyperphosphorylated tau and neurodegeneration. J. Neurochem 106, 107-120 (2008)

6. Cha-Molstad, H. et al. Amino-terminal arginylation targets endoplasmic reticulum chaperone BiP for autophagy through p62 binding. Nat. Cell Biol. 17, 917-929 (2015)

7. Cha-Molstad, H. et al. Regulation of autophagic proteolysis by the $\mathrm{N}$-recognin SQSTM1/p62 of the N-end rule pathway. Autophagy 14, 1-3 (2018).

8. Cha-Molstad, H. et al. p62/SQSTM1/Sequestosome-1 is an N-recognin of the $\mathrm{N}$-end rule pathway which modulates autophagosome biogenesis. Nat. Commun. 8, 102 (2017).

9. Varshavsky, A. The N-end rule. Cell 69, 725-735 (1992).

10. Sriram, S. M., Kim, B. Y. \& Kwon, Y. T. The N-end rule pathway: emerging functions and molecular principles of substrate recognition. Nat. Rev. Mol. Cell Biol. 12, 735-747 (2011).

11. Bachmair, A., Finley, D. \& Varshavsky, A. In vivo half-life of a protein is a function of its amino-terminal residue. Science 234, 179-186 (1986).

12. Kwon, Y. T., Xia, Z., Davydov, I. V., Lecker, S. H. \& Varshavsky, A. Construction and analysis of mouse strains lacking the ubiquitin ligase UBR1 (E3alpha) of the N-end rule pathway. Mol. Cell Biol. 21, 8007-8021 (2001).

13. Tasaki, T. et al. UBR box N-recognin-4 (UBR4), an N-recognin of the N-end rule pathway, and its role in yolk sac vascular development and autophagy. Proc. Natl Acad. Sci. USA 110, 3800-3805 (2013).

14. Choi, W. S. et al. Structural basis for the recognition of $\mathrm{N}$-end rule substrates by the UBR box of ubiquitin ligases. Nat. Struct. Mol. Biol. 17, 1175-1181 (2010).

15. Matta-Camacho, E., Kozlov, G., Li, F. F. \& Gehring, K. Structural basis of substrate recognition and specificity in the $\mathrm{N}$-end rule pathway. Nat. Struct. Mol. Biol. 17, 1182-1187 (2010).

16. Laplante, M. \& Sabatini, D. M. mTOR signaling in growth control and disease. Cell 149, 274-293 (2012).

17. Moscat, J., Karin, M. \& Diaz-Meco, M. T. p62 in cancer: signaling adaptor beyond autophagy. Cell 167, 606-609 (2016).

18. Duran, A. et al. p62 is a key regulator of nutrient sensing in the mTORC1 pathway. Mol. Cell 44, 134-146 (2011).

19. Linares, J. F. et al. Amino acid activation of mTORC1 by a PB1-domaindriven kinase complex cascade. Cell Rep. 12, 1339-1352 (2015).

20. Carroll, B. et al. Oxidation of SQSTM1/p62 mediates the link between redox state and protein homeostasis. Nat. Commun. 9, 256 (2018).

21. Ciuffa, R. et al. The selective autophagy receptor p62 forms a flexible filamentous helical scaffold. Cell Rep. 11, 748-758 (2015).

22. Gump, J. M. \& Thorburn, A. Sorting cells for basal and induced autophagic flux by quantitative ratiometric flow cytometry. Autophagy 10, 1327-1334 (2014).

23. Itakura, E. \& Mizushima, N. p62 Targeting to the autophagosome formation site requires self-oligomerization but not LC3 binding. J. Cell Biol. 192, 17-27 (2011).

24. Bjorkoy, G. et al. p62/SQSTM1 forms protein aggregates degraded by autophagy and has a protective effect on huntingtin-induced cell death. J. Cell Biol. 171, 603-614 (2005).

25. Paine, M. G., Bab, J. R., Seibenhener, M. L. \& Wooten, M. W. Evidence for p62 aggregate formation: role in cell survival. FEBS Lett 579, 5029-5034 (2005). 
26. Lamark, T. et al. Interaction codes within the family of mammalian Phox and Bemlp domain-containing proteins. J. Biol. Chem. 278, 34568-34581 (2003).

27. Wilson, M. I., Gill, D. J., Perisic, O., Quinn, M. T. \& Williams, R. L. PB1 domain-mediated heterodimerization in NADPH oxidase and signaling complexes of atypical protein kinase C with Par6 and p62. Mol. Cell 12, 39-50 (2003).

28. van der Zee, J. et al. Rare mutations in SQSTM1 modify susceptibility to frontotemporal lobar degeneration. Acta Neuropathol. 128, 397-410 (2014)

29. Xu, J. \& Van Doren, S. R. Tracking equilibrium and nonequilibrium shifts in data with TREND. Biophys. J. 112, 224-233 (2017).

30. Mi, W. et al. The ZZ-type zinc finger of ZZZ3 modulates the ATAC complexmediated histone acetylation and gene activation. Nat. Commun. 9, 3759 (2018).

31. Minor, W., Cymborowski, M., Otwinowski, Z. \& Chruszcz, M. HKL-3000: the integration of data reduction and structure solution--from diffraction images to an initial model in minutes. Acta Crystallogr. D. Biol. Crystallogr. 62, 859-866 (2006).

32. Emsley, P., Lohkamp, B., Scott, W. G. \& Cowstan, K. Features and development of Coot. Acta Crystallogr. D. Biol. Crystallogr. 66, 486-501 (2010).

33. Adams, P. D. et al. PHENIX: a comprehensive Python-based system for macromolecular structure solution. Acta Crystallogr. D. Biol. Crystallogr. 66, (213-221 (2010).

34. Allen, G. F., Toth, R., James, J. \& Ganley, I. G. Loss of iron triggers PINK1/ Parkin-independent mitophagy. EMBO Rep. 14, 1127-1135 (2013).

\section{Acknowledgements}

We thank Jay Nix at Beamline 4.2.2 of the ALS in Berkeley for help with X-ray crystallographic data collection and Ian Ganley for sharing the mCherry-GFP-Fis1 tandem construct. This work was supported by grants from NIH GM106416, GM101664 and GM100907 to T.G.K., CA192642 and CA218254 to M.T.D.-M., DK108743 and CA211794 to J.M., CA150925 and CA190170 to A.T., and CA204020 to X.S., and by the Basic Science Research Programs of the National Research Foundation funded by the MSIP of Korea (NRF-2016R1A2B3011389 to Y.T.K.), Protech Inc. internal fund to Y.T. K., and the Brain Korea 21 PLUS Program to Y.T.K.

\section{Author contributions}

Y.Z., S.R.M., J.F.L., J.W.A., C.G.T., C.H.J., B.E.F., M.R.H. and W. M. performed experiments and together with X.S., J.M., A.T., M.T.D-M., Y.T.K. and T.G.K. analyzed the data. Y.Z., M.T.D-M., Y.T.K. and T.G.K. wrote the manuscript with input from all authors.

\section{Additional information}

Supplementary Information accompanies this paper at https://doi.org/10.1038/s41467018-06878-8.

Competing interests: The authors declare no competing interests.

Reprints and permission information is available online at http://npg.nature.com/ reprintsandpermissions/

Publisher's note: Springer Nature remains neutral with regard to jurisdictional claims in published maps and institutional affiliations.

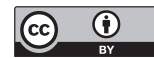

Open Access This article is licensed under a Creative Commons Attribution 4.0 International License, which permits use, sharing, adaptation, distribution and reproduction in any medium or format, as long as you give appropriate credit to the original author(s) and the source, provide a link to the Creative Commons license, and indicate if changes were made. The images or other third party material in this article are included in the article's Creative Commons license, unless indicated otherwise in a credit line to the material. If material is not included in the article's Creative Commons license and your intended use is not permitted by statutory regulation or exceeds the permitted use, you will need to obtain permission directly from the copyright holder. To view a copy of this license, visit http://creativecommons.org/ licenses/by/4.0/.

(C) The Author(s) 2018 